\title{
Seyfert Activity in Ring Galaxies due to Galactic Interactions
}

\author{
Tapan K. Chatterjee \\ Facultad de Ciencias, Fisico-Matematicas, Universidad A. Puebla, \\ Apartado Postal 1316, Puebla, Mexico.e-mail: tchat@mailexcite.com
}

Observations indicate that ring galaxies are more active than spirals (e.g., Ghigo et al., 1983 Appleton and Struck-Marcell, 1987). However, Seyfert activity is noted in only a few ring galaxies, e.g. NGC 985 (de Vaucouleurs \& de Vaucouleurs 1975), NGC $1144=$ Arp 118 (Huchra et al. 1982), ring galaxy in Sextans-optical counterpart of the IRAS source 09595-0755 (Wakamatus \& Nishida). This is indicative of the fact that this type of activity requires favorable circumstances. In this context a previous work on the stellar episode of ring formation, Chatterjee (1984), is extended, including gas and studying the evolution of the ring structure, due to rebounds of the compact elliptical about the plane of the disk.

The spiral galaxy (of radius $\mathrm{R}$ ) is modeled by an exponential disk of scale length $\alpha=4 / \mathrm{R}$, witha (static) thickness (Chatterjee 1990a), and a spherical polytropic bulge $(n=0.3 .4$ equally weighted combination) containing $1 / 3$ of the mass; about $20 \%$ of the mass of the disk contains gas particles. The disk is embedded in a static halo (of mass 5 times the optical parts) with an asymptotic dependence $\mathrm{M}(\mathrm{r}) \sim \mathrm{r}$ (c.f., Allen \& Martos 1986) as in one of the moddles of Chatterjee, 1990b. The elliptical is modeled identically as the bulge. The gravitational potential is softened with sofetning constants of $\epsilon=\mathrm{r}_{o} / 5, \mathrm{r}_{o} / 3$, $0.8 r_{o}, r_{o} / 4$ for the bulge of the spiral as well as the elliptical, stellar and gasous components of the disk, and for mutual gravitational interaction, respectively ( $\mathrm{r}_{0}$ being the radius containing $75 \%$ of the total mass of the spiral). The Lagrangian Equations (in polar cooridinates $\mathrm{r}$ and $\theta$ ) determine tyhe relative orbit of the elliptical (Galaxy e) with respect to the spiral (Galaxy s),

$$
\left.d t=\left[2 \mu^{-1}\left\{E-W(r)-L^{2} / 2 \mu r^{2}\right)\right\}\right]^{-0.5} d t, \text { and } d \theta=\left[L /\left(\mu r^{2}\right)\right] d t,
$$

where $\mu, \mathrm{L}$ and $\mathrm{E}$ are the reduced mass, angular momentum and the orbital energy, respectively, and $\mathrm{W}(\mathrm{r})$ is the instantatneious potential energy of interaction between the galaxies. At small intervals of $\mathrm{r}$ and $\theta$, the tital effects and the corresponding changes in velocities of the stars are determined, such that the instantaneous internal energy changes of the two galaxies $\Delta \mathrm{U}_{s}(\mathrm{t})$, can be determined; the instantaneous relative velocity follows from,

$$
V(t)=\left[2 \mu^{-1}\left\{E_{i}-W(t)-\Delta U_{e}(t)-\Delta U_{s}(t)\right\}\right]^{-0.5},
$$

$\mathrm{E}_{i}$ being the inital value of $\mathrm{E}$. The galaxies are interchanged at each interval to determine the relevant quantities for each one. The three-body approach is adopted to study the motion of the stellar and gasous particles, (but taking 
the extended nature of the particles into account through gravity softening). Marginally bound conditions are used so that rebounds of the spherical intruder occur; such that during the collision episode the perturber does not espcape abruptly but oscillates about the plane of the target disk.

Results indicate that after the formation of the stellar ring sturcture, the same propagates outwards, and then inwardws; such that it can be considered as a density wave with damped oscillatory behavior. The gas inner to the stellar ring, and leading it, experiences a clumping and decelerating torque, that causes the same to lose angular momentum and fall inward; while the gas outer to the ring gains angular momentum and moves outwards in expanding orbits. Though the ring disappears in due course of time, the same is reformed due to rebounds of the ellipti8cal intruder; such that we have a feedback process. Basically a bisymmetric potential develops due to the formation of the stellar ring; the same dissolves and redovelops, as the perturber oscillates about the plane of the disk, with growing amplitude. This process leads to a falttening of the surface density distribution and redistribution of the angular momentum of the gas in the disk. In this way the angular momentum exchange induces a turbulence in the gas content of the disk. This will render the ring galaxies more active than normal spirals, as is observed. The cumulative effects of the repeated oscillations of the intrudr is the accumulation of gas immediately surrounding the nucleus, in the form of an accretion disk. It is found that in the prescence of a deep potential well (corresponding, for example to that of a black hole), the gas will flow into the nucleus. As only several ring galaxies manifest Seyfert activity, this cound be indicative of the fact that Seyfert ring galaxies have compact central objects.

Acknowledgments. It is a pleasure to thank the organizers for an excellent hospitality

\section{References}

Allen, C., Martos, M.A., 1986, Rev. Mexicana. Astron, Astrof. 13, 137.

Appleton, P.N., Struck-Marcell, C., 1987, ApJ, 312, 566.

Chatterjee, T.K., 1984, Ap\&SS, 106, 309.

Chatterjee, T.K., 1990a, Ap\&SS, 163, 127.

Chatterjee, T.K., 1998b, IAU Symp. 124, "Paired and Interacting Galaxies" (NASA, USA).

de Vaucouleurs, G., de Vaucouleurs, Al., 1975, ApJ, 197, L1.

Ghigo, F.D., et al., 1983, AJ, 88, 1587

Huchra, J.P., Wyatt, W.F., Davis, M., 1982, AJ, 87, 1628.

Wakamatsu, K., Nishida, M.T., 1987, ApJ, 315, L23. 